\title{
Therapeutische Zügel am Lebensende lockern
}

\author{
Gleich alte Senioren können ganz unterschiedliche weitere Lebenserwartungen haben. \\ Dies sollte bei diagnostischen und therapeutischen Entscheidungen berücksichtigt werden.
}

\begin{abstract}
_ Vielen Fachgesellschaften empfehlen, die Lebenserwartung zu berücksichtigen. Zur Umsetzung in den USA wurden 28 Allgemeinärzte befragt, die zu etwa 25\% ältere Patienten betreuen. Die Ärzte waren im Mittel 46 Jahre alt und seit 16 Jahren mit der Ausbildung fertig.

Die Kollegen bestätigten, dass sie bei ärztlichen Entscheidungen die vermutete Lebenserwartung der Patienten für 5-10 Jahre berücksichtigen - vor allem bei lebensbegrenzenden Erkrankungen, bei multiplen, schweren oder chronischen Begleiterkrankungen und bei der Prävention. In erster Linie sind das Alter und der meist nur vage beurteilte Allgemeinzustand die bestimmenden Kriterien. Die Unsicherheit der Prognose führt dazu, dass auch alte Alte wie Erwachsene im mittleren Lebensalter be-
\end{abstract}

handelt werden. Einem rüstigen Ehepaar in den Achtzigern gibt man dann z. B. den Rat: „Sie leben bestimmt noch ein, zwei Jahrzehnte - da müssen wir bei der Vorbeugung etwas aggressiver sein.“"

- Schoenborn NL, Bowman II TL, Cayea D et al. Primary care
practitioners' views on incorporating long-term prognosis in the care of older adults. JAMA Intern Med. 2016;176:671-8

\section{KOMMENTAR}

Nach der amerikanischen Fachgesellschaft ADA sollen alte Diabetiker mit begrenzter Lebenserwartung nicht mehrt auf $\mathrm{HbA}_{1 c}$-Zielwerte unter $8 \%$ behandelt werden, weil das Risiko einer intensiven Behandlung den möglichen Nutzen überwiegt. Ähnliche Empfehlungen gibt es bei vielen Erkrankungen und in der Prävention.

Ebenso zahlreich wie verständlich sind die Gründe, Ursachen und Ausreden, die die Um- setzung solcher Empfehlungen verhindern. Jeder erfahrene Arzt hat erlebt, wie unsicher die Beurteilung der Lebensprognose im Einzelfall ist. Niedergelassene Ärzte sind es nicht gewohnt, mit zuverlässigen, aber meist komplizierten Scores zur Lebensprognose zu arbeiten.

Darüber hinaus verlangt das Selbstbestimmungsrecht des Patienten, dass auch solche Entscheidungen mit ihm besprochen werden. Das scheuen Ärzte, weil der Patient vermuten könnte, man "gebe ihn auf". Einige Ärzte befürchten auch aufwändigen und zeitraubenden Gesprächsbedarf oder juristische Konsequenzen.

Jahrzehnte lang soll man alles Erdenkliche für die Gesundheit tun - und im Alter soll das nicht mehr gelten? Ärzte und Patienten verstehen den Paradigmenwechsel oft nicht.

Prof. Dr. med. H. Holzgreve

\section{Akute Varikozele als Zeichen eines Nierentumors}

Ein 65-jähriger Mann suchte die urologische Ambulanz auf, weil er vor vier Wochen eine Schwellung im Bereich des linken Hodens bemerkt hatte. Die Untersuchung ergab eine vergrößerte variköse Vene, die sich bis in den Leistenkanal erstreckte (Abb. A). Zusätzlich tastete man eine Raumforderung im Bereich der linken Flanke. Im CT des Abdomens und des kleinen Beckens stellten sich mehrere Tumoren im Bereich beider Nieren dar (Abb. B), wobei der größte fast die gesamte linke Niere einnahm. Bei der offenen radikalen Nephrektomie der linken Niere wurde ein Nierenzellkarzinom diagnostiziert. Bereits eine Woche nach der Operation war die Varikozele verschwunden.

Bei der Varikozele handelt es sich um eine Dilatation des Plexus pampiniformis des Samenstrangs, die fast immer links auftritt. Das liegt daran, dass die linke V. spermatica fast rechtwinklig in die linke Nierenvene mündet. Dies sorgt für eine höhere Druckbelastung als bei der rechten V. spermatica, die etwas tiefer in einem spitzen Winkel direkt in die V. cava inf. mündet. Die meisten Varikozelen sind konstu- tionell-idiopathisch und entstehen langsam im jungen bis mittleren Lebensalter. Eine rasch aufgetretene Varikozele im höheren Lebensalter legt den Verdacht auf eine retroperitoneale Raumforderung nahe.

Prof. Dr. med. H. S. FüeßI

- El Abiad Y, Qarro A (yassineealabiad@gmail.com). Acute varicocele revealing renal cancer. NEngl J Med. 2016;374:2075
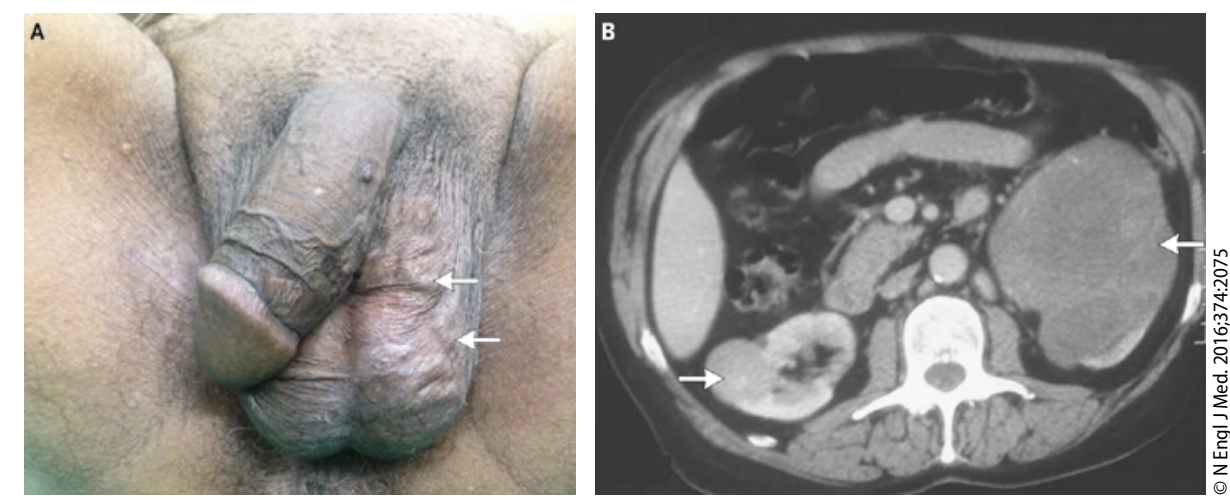

A: Variköse Vene am linken Hoden (Pfeile). B: Nierentumoren im CT (Pfeile). 